\title{
Generalised anxiety disorder
}

\author{
Christopher Gale, ${ }^{1}$ Oliver Davidson²
}

${ }^{1}$ Department of Psychological Medicine, Dunedin School of Medicine, University of Otago, PO Box 913, Dunedin, New Zealand, and Mental Health Services, Otago District Health Board, Dunedin

${ }^{2}$ Department of Psychological Medicine, Dunedin School of Medicine, University of Otago, PO Box 913, Dunedin, New Zealand, and Psychology Associates, Dunedin

Correspondence to: C Gale christopher.gale@stonebow. otago.ac.nz

BMJ 2007:334:579-81 doi: 10.1136/bmj.39133.559282.BE

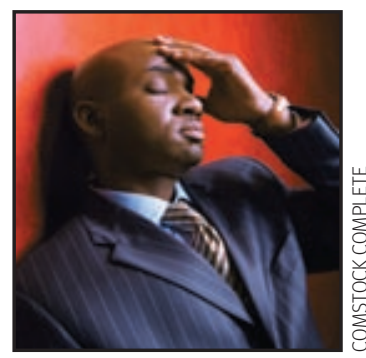

Generalised anxiety disorder is a syndrome of ongoing anxiety and worry about many events or thoughts that the patient generally recognises as excessive and inappropriate. However, the nature of "generalised worry" has been hard to describe in a categorical manner. The criteria required for making a diagnosis are evolving: these criteria clearly increase or decrease markedly the threshold for diagnosis. ${ }^{1}$

About $1-5 \%$ of the general population report having generalised anxiety disorder. Many of these people also have other disorders, and those with generalised anxiety disorder report a considerable level of disability. Long term follow-up studies suggest that generalised anxiety disorder is a condition that worsens the prognosis for any other condition, and that people who have only generalised anxiety disorder are likely to develop further conditions. The availability of and evidence for efficacious treatments has increased in the past five years.

\section{Sources and selection criteria}

We used the Clinical Evidence database ${ }^{2}$ then searched for community surveys, randomised controlled trials, and systematic reviews-using the term "generalised anxiety disorder"-in Medline, Embase, and the Cochrane Library up to June 2006.

\section{Who is likely to get generalised anxiety disorder?}

Most of the recent literature uses DSM-IV criteria for generalised anxiety disorder; the ICD-10 criteria place greater weight on somatic symptoms and explicitly limit comorbidity (box).

The table lists recent community surveys using DSMIV. These have shown that $1-5 \%$ of the population have reported generalised anxiety disorder in the past 12 months. The disorder is more common in women, and often occurs alongside mood disorders, anxiety disorders, somatoform disorders, and medical conditions. ${ }^{3-8}$

A review found that the rate of generalised anxiety disorder was significantly higher (odds ratio 3.3 (95\% confidence interval 2.0 to 5.5)) in those who had been invloved in civilian trauma (such as a dam collapse or toxic chemical spill). ${ }^{9}$ Reviews have linked the disorder with bullying (or peer victimisation) ${ }^{10}$ and an increase in the number of life events. ${ }^{11}$ Two reviews of family studies show an increased risk of the disorder in first degree relatives of patients. ${ }^{12} 13$

The incidence of generalised anxiety disorder in men

\author{
SUMMARY POINTS \\ Generalised anxiety disorder is a syndrome of ongoing \\ anxiety and worry about many events or thoughts that \\ the patient generally recognises as excessive and \\ inappropriate \\ Most people with generalised anxiety disorder also have \\ other mood and anxiety disorders \\ Several treatment efficacy trials have been conducted \\ but few effectiveness trials with generally representative \\ samples \\ Cognitive behaviour therapy is more efficacious than non- \\ directive psychotherapy or no treatment \\ Anxiety management treatment is also better than no \\ treatment and its efficacy may equal that of cognitive \\ behaviour therapy \\ Antidepressants, benzodiazepines, buspirone, and \\ kava are efficacious but often have clinically significant \\ adverse effects
}

is half that in women ${ }^{\mathrm{w} 1}$ and is lower in older people. ${ }^{\mathrm{w} 2} \mathrm{~A}$ review of 20 observational studies in younger and older adults suggested that autonomic arousal to stressful tasks was lower in older people and that older people became accustomed to stressful tasks more quickly than younger people. ${ }^{\text {w3 }}$

\section{How do people with the disorder pesent?}

Anyone presenting with a mood or anxiety disorder may have generalised anxiety disorder. Most screening questionnaires for the condition ask if the person is a worrier, if they worry overmuch about many things, and then ask if they have somatic symptoms of anxiety. As people with generalised anxiety disorder may develop other mood and anxiety syndromes over time, it is important to screen for these too, particularly depressive disorder.

\section{How can the effect of treatment be measured?}

In clinical trials the most commonly used clinician rating scale is the Hamilton anxiety scale, ${ }^{\mathrm{w} 4}$ a 14 item instrument that places an emphasis on somatic symptoms. The most used self report measures are the state trait anxiety inventory, ${ }^{\mathrm{w} 5}$ the Beck anxiety inventory, ${ }^{\mathrm{w} 6}$ and the Penn state worry questionnaire ${ }^{\mathrm{w} 7}$; the first and last of these four scales are in the public domain.

Treatment response is generally defined as a 50\% reduction in baseline score. Clinical recovery is often defined as a score of less than 7 on the Hamilton anxiety scale or a score of 1 or 2 on the clinical global impression scale. ${ }^{\text {w8 }}$ 
Prevalence of generalised anxiety disorder* in previous 12 months, and comorbidity, according to national community surveys. Values are percentages unless stated otherwise

\begin{tabular}{|c|c|c|c|c|c|c|}
\hline & Singapore3 & Germany4 & Australia5 & US4 & US5 & Europe6 \\
\hline Age (years) (No of participants) & $20-59(n=2847)$ & $18-64(n=7124)$ & $>18(n=10641)$ & $>18(\mathrm{n}=9282)$ & $>18(n=2657)$ & $>18(n=21425)$ \\
\hline Instrument & SCAN & M-CIDI & CIDI 2.1 & CIDI 3.0 & AUADIS & CIDI 3.0 \\
\hline Proportion of participants with GAD & 3.0 & 1.5 & 3.6 & 3.1 & 4.1 & 1.0 \\
\hline Proportion of men (women) & $1.0(3.6)$ & $1.0(2.1)$ & $3.2(4.0)$ & Not reported & $2.8(5.3)$ & $0.5(1.3)$ \\
\hline \multicolumn{7}{|l|}{ Of those with a diagnosis of GAD: } \\
\hline Proportion with any comorbidity & Not reported & 93.1 & 67.8 & 85 & & 69.4 \\
\hline Mood disorder & Not reported & 70.6 & 44.9 & Not reported & Not reported & Not reported \\
\hline Anxiety & Not reported & 55.9 & 37.4 & Not reported & Not reported & Not reported \\
\hline Somatoform & Not reported & 48.1 & Not reported & Not reported & Not reported & Not reported \\
\hline Substance misuse & Not reported & Not reported & 13.3 & Not reported & 23.0 & Not reported \\
\hline Medical condition & 53.9 & Not reported & Not reported & Not reported & Not reported & Not reported \\
\hline
\end{tabular}

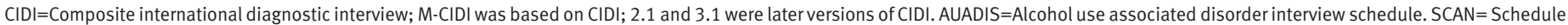
of clinical assessment in neuropsychiatry. *As diagnosed according to the DSM-IV criteria.

\section{What is the outcome for patients?}

Evidence on long term prognosis is sparse. At 12-year follow-up of adults at an anxiety clinic, $42 \%$ of patients had recovered from generalised anxiety disorder, but the disorder was a marker for poor outcome for those patients who had another anxiety disorder. ${ }^{14}$ In a similar cohort of 68 people with generalised anxiety disorder alone (as defined by DSM-III criteria) at initial assessment and followed over 12 years, two had the disorder alone after 12 years, 28 no longer had a diagnosis, 12 had developed dysthymic disorder, and 10 had developed depression; the rest had been lost to follow up. ${ }^{15}$

\section{Which psychological treatments can help?}

Both cognitive therapy and anxiety management therapy are efficacious, and cognitive behaviour therapy may be more efficacious than anxiety management therapy alone.

Anxiety management therapy is a structured therapy involving education, relaxation training, and exposure but does not include cognitive restructuring; cognitive behaviour therapy adds to this a cognitive restructuring element. Relaxation involves practising techniques that lead to muscular or bodily relaxation. Exposure entails (over a period of time) graded, repeated confrontation (through visualisation, image, or the stimulus) with a stimulus that

\section{A patient's perspective}

As a child, I was excessively worried and nervous. I tended to over-analyse situations, was fidgety, and found it difficult to relax. At age 18 , I had my first intense anxiety experience, after which my anxiety became significantly worse. Fearful of social and physical situations, I avoided potential anxious situations and often used alcohol to deal with social situations. My anxieties could change overnight and manifest into a seemingly unsolvable problem. I often worried about my mental state and felt I had to hide my emotions and thoughts from strangers, friends, family, and doctors.

I forced myself to talk to my general practitioner as my anxiety would not subside. For over two years I used medication, which helped significantly. However, I also wished to seek psychological advice to understand what the future might hold for me. I was told by a clinical psychologist that with the right tools and training I would probably be able to change my thought patterns, which had became irrational and negative, and my coping behaviour. It was a great relief to know there could be a better future.

Understanding the errors in my thinking and implementing better coping strategies has reduced my anxiety levels considerably. And this has therefore enabled me to live a much more balanced and normal life.

Martin, aged 37 causes anxiety. Cognitive restructuring involves challenging the dysfunctional thought processes and the underlying assumptions that may be related to the symptoms.

Systematic reviews and subsequent randomised trials found that cognitive behaviour therapy significantly improved anxiety and depression over four to 12 weeks compared with the waiting list control group, anxiety management alone, relaxation alone, or non-directive psychotherapy. ${ }^{16} 17$ w9-w11 Patients randomised to anxiety management therapy also fare better than waiting list controls, and the efficacy of this treatment may equal that of cognitive therapy. ${ }^{2}$ w12 w13

\section{Which drug treatments can help?}

Two systematic reviews found that antidepressants (imipramine, paroxetine, and venlafaxine) improved symptoms over four to 28 weeks compared with placebo..$^{19} 19$ Clinical trials have found no significant differences among clinical responses to these antidepressants ${ }^{\mathrm{w} 14}$ or between antidepressants and benzodiazepines ${ }^{\mathrm{w} 15}$ or antidepressants and buspirone. ${ }^{w 16}$ In a systematic review buspirone improved symptoms over four to nine weeks compared with placebo. ${ }^{20}$ One systematic review found that benzodiazepines reduced symptoms over two to nine weeks compared with placebo. ${ }^{21}$ A clinical trial found no significant difference in symptoms over three to eight weeks between benzodiazepines, between benzodiazepines and buspirone, hydroxyzine, or abecarnil (not available in the United Kingdom or New Zealand). ${ }^{\text {w17 }}$ w18 In a systematic review Kava extract significantly reduced symptoms compared with placebo (according to scores on the Hamilton anxiety scale). ${ }^{21}$ There have been some case reports, however, of severe hepatic compromise in patients receiving kava. ${ }^{\text {w19 }}{ }^{\mathrm{w} 20}$ Evidence from clinical trials indicates that hydroxyzine $^{\text {w21 w20 }}$ and pregabalin ${ }^{\text {w23 }}$ may be efficacious.

\section{What further research should be done?}

The evidence base for generalised anxiety disorder has grown in recent years. The development of standard methods for conducting and reporting such trials means that the newer trials are of a higher quality and are reasonably comparable.

There are still, however, few trials of clinical effectiveness. More are needed because most patients with gen- 


\section{ADDITIONAL EDUCATIONAL RESOURCES}

Resources for health professionals

- Treatment Protocol Project. Management of mental disorders. 4th ed. Darlinghurst, NSW: World Health Organization Collaborating Centre for Evidence in Mental Health Policy, 2004. - Clinical Research Unit for Anxiety and Depression www.crufad.com/cru_index.htm Contains information on anxiety plus a computerised intervention, which currently needs to be prescribed by a general practitioner

- British Association for Behavioural and Cognitive Psychotherapies www.babcp.com - Has a list of therapists, training resources, and general information for patients about anxiety and cognitive behaviour therapy

- Gale C. Generalised anxiety disorder. Clin Evidence. www.clinicalevidence.com/ceweb

conditions/meh/1002/1002.jsp

\section{RESOURCES FOR PATIENTS}

- Bourne E. The anxiety and phobia workbook. 4th ed. Oakland, CA: New Harbinger, 2005. (For use as a supplement for anxiety management training.)

\section{Current diagnostic criteria for generalised anxiety disorder}

\section{Diagnostic and statistical manual of mental disorders (DSM-IV-TR)}

- Excessive anxiety and worry (apprehensive expectation), occurring more days than not for at least six months, about a number of events or activities (such as work or school performance)

- The person finds it difficult to control the worry

- The anxiety and worry are associated with three or more (only one for children) of the following six symptoms, with at least some symptoms present for more days than not for the past six months): restlessness or feeling keyed up or on edge; being easily fatigued; difficulty concentrating or mind going blank; irritability; muscle tension; and sleep disturbance

- Anxiety and worry owing to panic disorder, social phobia, obsessive compulsive disorder, and separation anxiety disorder are excluded

International statistical classification of disease and related health problems, 10th revision (ICD-10)

The patient must have experienced at least six months with predominant tension, worry, and feelings of apprehension about everyday events and problems. At least four of the symptoms below must be present (at least one of which from the first group)

\section{Autonomic arousal symptoms}

Palpitations or pounding heart, or accelerated heart rate; sweating; trembling or shaking; dry mouth (not due to medication or dehydration)

Symptoms involving chest and abdomen

Difficulty breathing, feeling of choking, chest pain or discomfort, nausea or abdominal distress (such as churning in stomach)

\section{Symptoms involving mental state}

Feeling dizzy, unsteady, faint, or light-headed; feeling that objects are unreal (derealisation) or that the self is "not really here" (depersonalisation); a feeling of losing control, "going crazy," or passing out; fear of dying

\section{General symptoms}

Hot flushes or cold chills; numbness or tingling sensations; muscle tension or aches and pains; restlessness and inability to relax; feeling keyed up, on edge, or mentally tense; a sensation of a lump in the throat or difficulty in swallowing

\section{Other non-specific symptoms}

Exaggerated response to minor surprises or being startled; difficulty in concentrating or mind "going blank" because of worrying or anxiety; persistent irritability; difficulty in getting to sleep because of worrying

Panic disorder, phobic anxiety disorder, obsessive-compulsive disorder, or hypochondrical disorder criteria must not be met. If the symptoms are due to a physical disorder or organic mental condition or a substance related disorder, generalised anxiety disorder is excluded

eralised anxiety disorder have other mood and anxiety disorders too and are affected for a prolonged period ${ }^{14}$ and because the nature of comorbid conditions can change over time. ${ }^{15}$ These trials should compare the following: the efficacious psychotherapies; the efficacious medications; and psychotherapies versus medications. Any such trial design should be sufficiently powered to allow for analysis of comorbid conditions and be designed to run over a longer period of time than previous trials. Furthermore, some treatment options such as benzodiazepines should be examined by meta-analysis of efficacy before further trials are considered.

We thank our patient, Martin (see "A patient's perspective" box), for his description of his generalised anxiety disorder.

Contributors: CG was involved in the development of the search strategy, the resource selection, and the drafting of the paper; he is also the guarantor. OD helped with resource collection, the patient description, and drafting the paper. Kate Thompson helped to develop the search strategy. Keren Skegg and Richard Mullen reviewed the paper before publication.

Competing interests: CG has given talks for Lilly Pharmaceuticals and has attended conferences paid for by Lilly and Jannsen Pharmaceuticals. He has no shares, has not been a consultant to, or an investigator in clinical trials funded by, any pharmaceutical company.

Provenance and peer review: Commissioned and peer reviewed.

1 Slade T, Andrews G. DSM-IV and ICD-10 generalized anxiety disorder: discrepant diagnoses and associated disability. SocPsychiatry Psychiatr Epidemiol 2001;36(1):45-51.

2 Gale C. Generalised anxiety disorder. Clin Evidence. www.

clinicalevidence.com/ceweb/conditions/meh/1002/1002.jsp

$3 \mathrm{Lim}$ L, Ng TP, Chua HC, Chiam PC, Won V, Lee T, et al. Generalised anxiety disorder in Singapore: prevalence, co-morbidity and risk factors in a multiethnic population. Soc Psychiatry Psychiatr Epidemiol 2005;40:972-9.

4 Carter RM, Wittchen HU, Pfister H, Kessler RC. One year prevalence of subthreshold and threshold DSM-IV generalized anxiety disorder in a nationally representative sample. Depress Anxiety 2001;13:78-88.

5 Hunt C, Issakidis C, Andrews G. DSM-IV generalized anxiety disorder in the Australian national survey of mental health and well-being. Psychol Med 2002;32;649-59.

6 Kessler R, Chui W, Demler O, Walters E. Prevalence, severity and comorbidity of 12-month DSM-IV disorders in the national comorbidity survey replication. Arch Gen Psychiatry 2005;62:617-27.

7 Conway K, Compton W, Stinson F, Grant B. Lifetime comorbidity of DSM-IV mood and anxiety disorders and specific drug use disorder: results from the national epidemiologic survey of alcohol and related conditions. J Clin Psychiatry 2006;67:247-57.

8 Alonso J, Angermeyer MC, BernertS, Bruffaerts R, Brugha TS, Bryson H, et al. 12-Month comorbidity patterns and associated factors in Europe: results from the European Study of the Epidemiology of Mental Disorders (ESEMeD) project. Acta Psychiatr Scand Suppl 2004;420:28-37.

9 Brown ES, Fulton MK, Wilkeson A, Petty F. The psychiatric sequelae of civilian trauma. Compr Psychiatry 2000;41:19-23.

10 Hawker DSJ, Boulton MJ. Twenty years' research on peer victimisation and psychosocial maladjustment: a meta-analytic review of cross-sectional studies. I Child Psychol Psychiatr 2000;41:441-5.

11 Seivewright N, Tyrer P, Ferguson B, Murphy S, Johnson T. Longitudinal study of the influence of life events and personality status on diagnostic change in three neurotic disorders. Depress Anxiety 2000;11:105-13.

12 MiddeldorfCM, Cath CD, van Dyck R, Boomsa DI. The co-morbidity of anxiety and depression in the perspective of genetic epidemiology: A review of twin and family studies. Psychol Med 2005;35:611-24.

13 Hettema JM, Neale MC, Kendler KS. A review and meta-analysis of the genetic epidemiology of anxiety disorders. Am J Psychiatry 2001;158:1568-78.

14 Bruce SE, Yonkers KA, Otto MW, Eisen JL, Weisberg RB, Pagano M, et al. Influence of psychiatric comorbidity on recovery and recurrence in generalized anxiety disorder, social phobia, and panic disorder: a 12-year prospective study. Am J Psychiatry 2005;162:1179-87.

15 Tyrer P, Seivewright $H$, Johnson T. The Nottingham study of neurotic disorder: predictors of 12-year outcome of dysthymic, panic and generalized anxiety disorder. Psychol Med 2004;34:1385-94.

16 Gould RA, Otto MW, Pollack MH, Yap L. Cognitive behavioural and pharmacological treatment of generalized anxiety disorder: a preliminary meta-analysis. Behavior Therapy 1997;28:285-305.

17 Westen D, Morrison K. A multidimensional meta-analysis of treatments for depression, panic and generalized anxiety disorder: an empirical examination of the status of empirically supported therapies. J Consult Clin Psychol 2001;69:875-89.

18 Mitte K, Noack P, Steil R, Hautzinger M. A meta-analytic review of the efficacy of drug treatment in generalized anxiety disorder. J Clin Psychopharmacol 2005;25:141-50.

19 Kapczinski F, Lima MS, Souza JS, Cunha A, Schmitt R. Antidepressants for generalized anxiety disorder. Cochrane Database Syst Rev 2003;(2): CD003592.

20 Gammans RE, Stringfellow JC, Hvisdos AJ, Seidehamel RJ, Cohn JB, Wilcox CS, et al. Use of buspirone in patients with generalized anxiety disorder and coexisting depressive symptoms: a meta-analysis of eight randomized, controlled studies. Neuropsychobiology 1992;25:193-201.

21 Pittler M, Ernst E. Efficacy of kava extract for treating anxiety: systematic review and meta-analysis. J Clin Psychopharmacology 2000;20(1):84-9. 